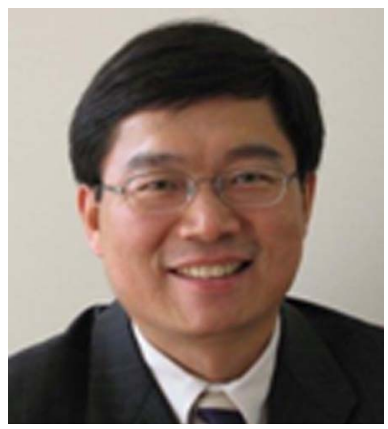

\section{From the New Editor-in-Chief}

It is an honor and pleasure to have been appointed as the Editor-in-Chief of the Journal of Biomedical Optics. Please allow me to thank my predecessor, Bruce Tromberg, and the editorial board as well as the JBO staff for their tremendous vision and unparalleled efforts to make this journal a great success. JBO is also deeply indebted to all of its authors, reviewers, and readers for their enthusiastic support.

Our greatest challenge is to improve a journal that is already a flagship in the growing field of biomedical optics. Journal publishing in today's competitive environment is analogous to sailing upstream; a journal must advance, or it will lag behind. To maintain the momentum of JBO, we are evaluating the following ten initiatives and, if feasible, plan to institute them as soon as logistics permit. The first five are intended to minimize the publication time, the next three to encourage submission of the highest-quality work, and the final two to improve delivery.

1. For JBO letters submissions, the review cycle will be targeted for " $1+1+10$ " days. I will assign the received manuscript to an editorial board member within 24 hours on average. The editorial board member will strive to identify potential reviewers within the next 24 hours. Reviewers will be requested to complete their initial reviews within 10 days.

2. All accepted JBO letters will be published online in professionally copyedited and typeset form in one month or less. Immediate publication in preprint form may also be a possibility in the future. In addition, SPIE has graciously granted all JBO letters immediate open access online. The JBO letters section, I believe, will boast the speed and accessibility of an online-only express journal as well as the quality of a conventional letters journal.

3. For full-length manuscripts, the review cycle will be correspondingly aimed at " $2+2+20$ " days. Full-length articles will continue to be the backbone of JBO.

4. A special reviewer database of young or new scientists holding doctoral degrees-such as postdoctoral fellows, assistant professors, and anyone new to the field-will be developed, while the general database also will continue to be expanded. I invite interested candidates to submit contact and expertise information to JBO via jbo@spie.org.

5. Authors will be further encouraged to suggest editorial board members and reviewers to manage and review their manuscripts. Such suggestions are often adopted, at least in part.

6. The editorial board will actively solicit experts to submit reviews, tutorials, and outlooks. Some of these articles will enjoy immediate open access online.

7. We will look into having a JBO Best Paper Award to be presented annually at the SPIE Biomedical Optics Symposium at Photonics West.

8. For those who attend the Biomedical Optics Symposium, we will experiment with directing manuscripts that authors deem ready for peer review to JBO, as an alternative submission option for conference authors.

9. Due to the growing volume of submissions and published papers, publication will transition from bimonthly to monthly, possibly in 2011.

10. For readability of the tables of contents of each issue, fulllength articles will be categorized into the following four areas: General, Imaging, Sensing, and Therapeutic (GIST). Authors will select a category when submitting manuscripts.

Finally, I request all of our contributors to continue supporting JBO. Any suggestions on improving the journal would be greatly appreciated. The editorial board, the JBO staff, and I all look forward to serving our community. 